\title{
Schwanoma retroperitoneal maligno
}

\author{
R. Ferrero Doria, B. Coronel Sánchez, E. Huertas Valero*, F. García Víctor, M. Gassó Matoses, \\ E. Díaz Calleja
}

Servicio de Urología. *Servicio de Anatomía Patológica. Hospital Francesc Borja. Gandia (Valencia).

Actas Urol Esp 2005; 29 (4): 416-418

\section{RESUMEN}

\section{SCHWANOMA RETROPERITONEAL MALIGNO}

Presentamos un caso infrecuente de Schwanoma retroperitoneal maligno de diagnostico casual. Describimos sus manifestaciones clínicas, exploraciones diagnósticas, y el tratamiento utilizado.

Palabras clave: Tumor retroperitoneal. Schwanoma maligno. Neurilemoma.

\section{ABSTRACT \\ MALIGNANT RETROPERITONEAL SCHWANNOMA}

We present an uncommon case of retroperitoneal malignant schwannoma diagnosed accidentally. The clinical, diagnostic and therapeutic features are discussed.

Keywords: Retroperitoneal tumor. Malignant Schwannoma. Neurilemoma.

$\mathrm{L}$ os tumores que asientan en el retroperitoneo suelen ser poco frecuentes y se suelen clasificar según el tejido del que se originan. Dentro de los tumores derivados de las vainas nerviosas se encuentra el Schwanoma, considerándose que supone entre 1 - 10\% del total de neoplasias primarias retroperitoneales ${ }^{1}$. En el presente caso clínico presentamos un Schwanoma maligno diagnosticado de forma accidental, como suele ocurrir en este tipo de cánceres, tras la realización de una nefrectomía radical izquierda.

\section{CASO CLÍNICo}

V.P.C. Varón de 66 años con antecedentes de hipercolesterolemia, HTA, exfumador desde hace 4 años y disfunción eréctil. Ingresa a través del Servicio de Urgencias por deterioro del estado general. La exploración es anodina y no se observan ningún estigma cutáneo significativo. Tras ecografía abdominal se observa masa hipoecogénica heterogénea de polo superior de riñón izquierdo. El TAC muestra masa de contornos polilobulados e hipodensos, de unos $11 \mathrm{~cm}$ de diámetro y situada en el polo superior de riñón izquierdo (Fig. 1). Se observa afectación de la grasa perirrenal pero no adenopatías locorregionales. Los estudios analíticos sanguíneos y de orina fueron normales.

Se realiza Nefrectomía renal izquierda ampliada por vía anterior, extrayéndose una masa de tamaño considerable.

El estudio anatomopatológico describe un tumor cuyas dimensiones son de 12,5 x 9,5 x 9,5 cm, que invade la cápsula renal pero no el parénquima ni la vía excretora del citado riñón. A microscopio se observan células fusiformes de borde impreciso dispuestas en fascículos y con cierto pleomorfismo nuclear. El estudio inmunohistoquímico es positivo a Vimentina S-100, así como a Enolasa neuroespecífica, todo ello compatible con Schwanoma maligno, que en este caso se localiza en el retroperitoneo (Fig. 2).

Durante el seguimiento el paciente ha presentado un ACV por infarto isquémico temporal derecho sin apenas secuelas. En un TAC de control a los 2 años, se aprecia un engrosamiento de pared anterior gástrica que tras endoscopia digestiva alta y biopsia, se diagnostica de adenocarcinoma gástrico, siendo sometido, posteriormente, a gastrectomía subtotal. 


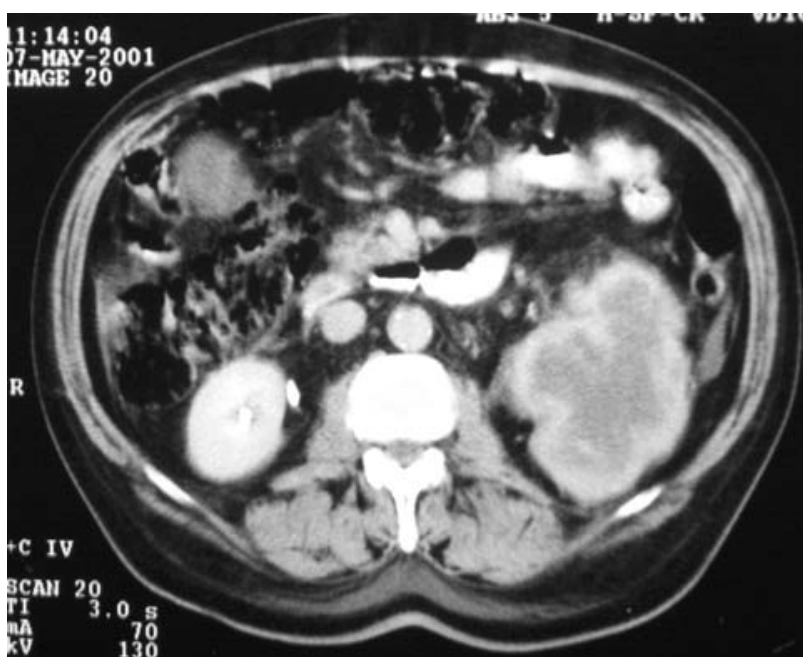

FIGURA 1. TAC abdominal: masa en fosa fosa renal izquierda.

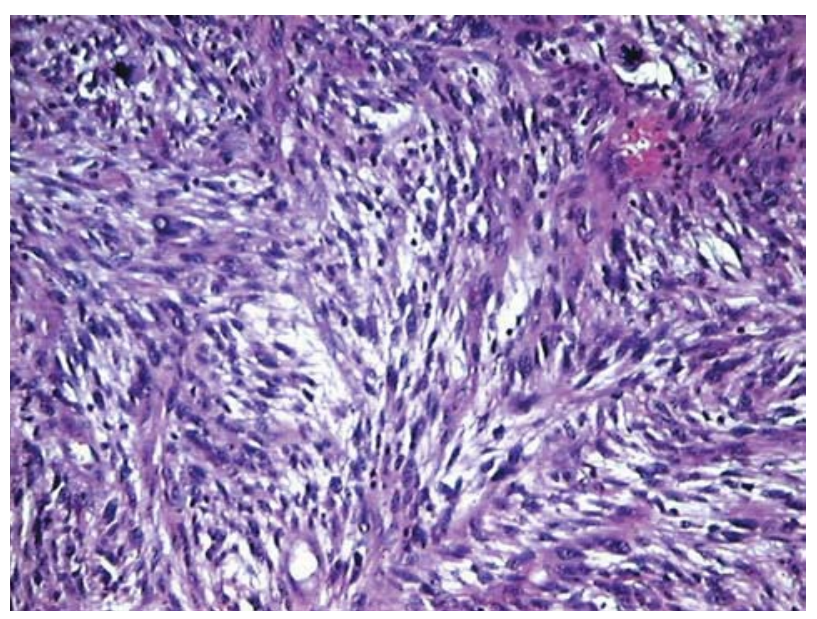

FIGURA 2. Anatomía patológica del Schwanoma maligno.

A los cuatro años de la nefrectomía, y tras controles con TAC, el paciente no presenta, hasta el momento, enfermedad residual en el lecho quirúrgico ni enfermedad metastásica, estando libre de enfermedad.

\section{DISCUSIÓN}

En 1954 Ackerman $^{2}$ clasifica los tumores retroperitoneales primarios atendiéndose a los hallazgos histológicos y los divide en: los tumores de origen nervioso, los de origen mesodérmico y los originados a partir de restos embrionarios. El schwanoma maligno es un tumor raro, proveniente de las células de Schwann, las cuales constituyen el tejido de soporte de los nervios periféricos. También ha sido designado como sarcoma neurogénico o neurofibrosarcoma o neuri- lemoma maligno ${ }^{3}$. Esta variedad de Schwanoma fue descrita por primera vez en 1932 por Masson (referenciado por Romics) ${ }^{4}$. El $3 \%$ de los neurilemomas malignos se desarrollan en el retroperitoneo, pudiendo presentarse aislados o asociados a la enfermedad de Von Recklinghausen ${ }^{5}$. En nuestro caso tenemos dudas sobre el origen exacto de la tumoración dado que, como indica la descripción anatomopatológica, invade la cápsula renal, creándonos dudas sobre si se origina de la misma o ésta se ve afectada por crecimiento del tumor y más siendo como es que Romics ${ }^{4}$ describe un caso cuyo origen es la cápsula renal.

Suelen afectar de forma indiferente a varón o a mujer y su periodo de más elevada incidencia está entre los 40 y 60 años ${ }^{3,6}$.

Los síntomas con los que se ponen de manifiesto suelen ser consecuencia de la ocupación de espacio a que son acreedores, con manifestaciones de origen digestivo. También pueden mostrarse con anemia, fiebre, dolor en flanco, masa palpable, pérdida de peso $^{4,7}$. Frecuentemente van asociados a la enfermedad de Von Recklinghausen, siendo en estos casos de curso clínico diferente y de peor pronóstico ${ }^{3}$. La localización del Schwanoma también va a ser determinante a la hora de producir síntomas, habiéndose descrito en riñón, perineo, psoas, vaginal testicular, cavidad pelviana, cordón espermático ${ }^{3,8}$.

El diagnóstico preoperatorio rara vez es posible y son el TAC o la ecografia las exploraciones que diagnostican una masa que posteriormente será sometida a intervención quirúrgica. Algunos recurren a la angiografía para estudiar la vascularización y la operabilidad de la masa ${ }^{9}$. El estudio urográfico puede ser útil para valorar la repercusión que tiene la masa retroperitoneal sobre la vía urinaria.

La intervención quirúrgica completa suele ser el tratamiento más correcto, siendo necesaria la extirpación de algunos órganos vecinos en ciertas ocasiones, así como la resección de adecuados márgenes quirúrgicos ${ }^{1,10}$. Posteriormente al tratamiento han sido probados fármacos quimioterápicos sin conseguirse con ello mejoría en la supervivencia ${ }^{3}$. La radioterapia prácticamente carece de indicación ${ }^{7}$.

El Schwanoma maligno suele ser una neoplasia con tendencia a la recurrencia y metástasis, 
con un pronóstico oscuro, siendo la supervivencia del 23-30\% a los 5 años si está asociada a la enfermedad de Von Recklinghausen y del 45-75\% si no lo está ${ }^{3}$.

El diagnóstico de esta estirpe patológica se realiza ya en el postoperatorio tras el estudio de la pieza quirúrgica, en la que se observan células fusiformes de bordes imprecisos, dispuestas en fascículos y con pleomorfismo nuclear. Las técnicas inmunohistoquímicas revelan Vimentina S-100 positiva, así como Enolasa neuroespecifica también positiva.

Los Schwanomas malignos retroperitoneales suelen caracterizarse por ser muy poco frecuentes, diagnosticarse como masa accidental tras el estudio de sintomas propios de un sindrome ocupante de espacio, siendo objetivados con ecografia o TAC. Tras la extirpación quirúrgica se reconoce esta extirpe anatomopatológica, siendo el pronóstico habitualmente infausto.

\section{REFERENCIAS}

1. Hurley L, Smith JJ, Larsen C, Silverman M: Múltiple retroperitoneal schwannomas: case report and review of the literature. J Urol 1994; Feb151,413-416.

2. Ackerman LV. Tumors of the retroperitoneal mesentery and peritoneum. En: Atlas of tumor pathology. Washington, DC: Armed Forces Institute of Pathology, National Research Council, 1954;11,23-24:136.
3. González Enguita C, Caro Cebrián C, Gil Sanz MJ, et al: Obstrucción del tracto urinario superior causada por un Schwannoma maligno retrovesical. Estrategia terapéutica. Actas Urol Esp 1990; 14(1):46-49.

4. Romics I, Bach D, Beutler W. Malignant Schwannoma of kidney capsule. Urology 1992; Nov;40 (5):453-455.

5. Sordillo PP, Helson L, Hajdu SI, Magill GB, Kosloff C, Golbey RB, Beattie EJ. Malignant Schwannoma - clinical characteristics, survival and response to therapy. Cancer 1981;May 15;47(10):2503-2509.

6. Rodríguez Tolrá J, Sanfeliu Cortés F, Pujol Bosch F, Chavarria Mendoza J, Serrallach Milá N. Tumor retroperitoneal infrecuente: Schwanoma. A propósito de un caso. Actas Urol Esp 1982; Mar-Apr;6(2):99-102.

7. Feraro M, Arcari F, Federico R. Su di un raro caso di tumore retroperitoneale: lo schwanoma maligno. Min Chir 1984; 39:549.

8. Navalón P, Picurelli L, Ferrer Jiménez R, Tarín M, Bataller J, Ferrer Roda J. Schwannoma retroperitoneal: aportación de un caso. Arch Esp de Urol, 1989;May;42(4):366-369.

9. Fabbro MA, Costa L, D`Agostino S, Musi L, Belloli GP. Justa-adrenal malignant schwannoma. Pediatr Surg Int 1997; Sep;12(7): 532-534.

10. Guz B, Wood DJr, Mntie J, Pontes E. Retroperitoneal neural sheath tumores: Cleveland Clinic experience. J. Urol 1989;Dec;142 (6): 1434-1437.

Dr. R. Ferrero Doria

Avda. Albaida nº 2, esc. 1, pta. 18.

46870 Ontinyent (Valencia)

(Trabajo recibido el 17 septiembre 2004) 